\title{
Benign vascular sexual headache and exertional headache: interrelationships and long term prognosis
}

\author{
Peter L Silbert, Robert H Edis, Edward G Stewart-Wynne, Sasson S Gubbay
}

\begin{abstract}
There is a definite relationship between the vascular type of benign sexual headache and benign exertional headache. Forty five patients with benign vascular sexual headache were reviewed. Twenty seven $(60 \%)$ experienced benign vascular sexual headache alone and eighteen $(40 \%)$ had experienced both benign vascular sexual headache and benign exertional headache on at least one occasion. The mean age was 34.3 years with a male:female ratio of $5 \cdot 4: 1$. thirty patients with a history of benign vascular sexual headache were followed for an average of 74 months. A personal history of migraine was found in $47 \%$ of cases and a family history of migraine in $30 \%$. Forty one per cent of patients with benign vascular sexual headache alone had recurrences after diagnosis, and stress and fatigue were considered major contributing factors to the initial and recurrent headache. Nine patients had experienced benign vascular sexual headache and benign exertional headache within 72 hours of each other on at least one occasion, often with a residual headache between the two. Four patients experienced their benign vascular sexual headache and benign exertional headache separated by months to years. The prognosis of benign vascular sexual headache and the clinical and possible pathophysiological relationships between benign vascular sexual headache and benign exertional headache are discussed. Knowledge of the interrelationships of these varieties of headache is valuable in the counselling of patients.
\end{abstract}

Since the original description by $\mathrm{Kriz}^{1}$ in 1970 , benign sexual headache has become a well defined entity with three types being described according to presumed clinical and pathophysiological mechanisms. ${ }^{23}$ Type 1 has been equated with a muscle contraction headache developing before orgasm and associated with tensing of shoulder and neck musculature, and facial grimacing. Type 2 is the vascular type of sexual headache starting suddenly at the time of orgasm and thought to be related to the haemodynamic changes that occur at this time. Type 3 is the rarer low CSF pressure headache due to a dural tear. Whilst the prognosis for type 3 sexual headache is well understood and explicable on pathophysiological grounds, the prognosis of
Type 1 and 2 sexual headache remains unclear, with only Lance's original study having addressed this issue. ${ }^{2}$

Benign exertional headache in the form of sudden onset of a generalised headache, during any form of exertion has been described in patients with sexual headache, ${ }^{2-6}$ although any apparent association between vascular sexual headache and exertional headache has been the subject of conjecture. This study aimed to evaluate the interrelationships between benign vascular sexual headache and benign exertional headache as well as determining the prognosis for patients who experience benign vascular sexual headache (type 2 ).

\section{Method}

Patients previously evaluated by the authors in whom a diagnosis of benign vascular sexual headache (Type 2) had been made were included in the study. Benign vascular sexual headache was defined as the sudden onset of a throbbing or a constant headache during sexual activity, at or around the time of orgasm, (in whom other causes such as subarachnoid haemorrhage were excluded or considered unlikely according to history, examination, and at times, special investigation. Benign masturbatory cephalgia was excluded).

Attempts were made to follow up and interview all patients. Patients unable to be followed up were included in the data used to calculate the percentage of patients with benign vascular sexual headache who also experienced benign exertional headache, and the age and sex distribution at presentation only. The original case records were reviewed and this information in combination with that obtained at the follow up review was used to record age at onset of headache, sex, type of sexual headache, personal or immediate family history of migraine (Definition ${ }^{7}$ ), and other headaches including exertional headaches (defined as sudden onset of a "benign" sustained headache during any form of exertion, intentionally distinguishing it from certain strain or valsalva headaches where there is a short lived exacerbation of a pre-existing headache). Also recorded were months of follow up (from time of initial assessment rather than initial headache); number of attacks before presentation; time to diagnosis; circumstances of recurrence; possible predisposing factors for initial and any recurrent headache; investigations performed; presence of other medical illnesses before and after initial assessment. 
Table 1a Age and sex distribution of all patients with benign vascular sexual headache

\begin{tabular}{lllll}
\hline All patients & Number & $\begin{array}{l}\text { Mean }(S D) \text { age } \\
\text { years }\end{array}$ & $\begin{array}{l}\text { Age range } \\
\text { years }\end{array}$ & M:F ratio \\
\hline BVSH+/-BEH & 45 & $34 \cdot 3(9 \cdot 3)$ & $19-58$ & $5 \cdot 4: 1$ \\
BVSH alone & 27 & $36 \cdot 4(9 \cdot 4)$ & $20-58$ & $8: 1$ \\
BVSH/BEH (close) & 11 & $32 \cdot 1(7 \cdot 6)$ & $23-50$ & $4 \cdot 5: 1$ \\
BVSH/BEH (remote) & 7 & $29 \cdot 6(10 \cdot 0)$ & $19-41$ & $2 \cdot 5: 1$ \\
Table 1b Age and sex distribution of review cases & & & \\
Review cases & Number & Mean $(S D)$ age & $\begin{array}{l}\text { Age range } \\
\text { years }\end{array}$ & M:F ratio \\
\hline BVSH alone & 17 & $37 \cdot 1(9 \cdot 3)$ & $26-58$ & $7 \cdot 5: 1$ \\
BVSH/BEH (close) & 9 & $33 \cdot 9(7 \cdot 2)$ & $26-50$ & $3 \cdot 5: 1$ \\
BVSH/BEH (res) & 4 & $27 \cdot 2(9 \cdot 5)$ & $20-41$ & $3: 1$ \\
BVSH/BEH (remote) & 4 &
\end{tabular}

Legend: BVSH = benign vascular sexual headache, BEH = benign exertional headache, BVSH/BEH (close) = "close" benign vascular sexual and exertional headache and BVSH/BEH (remote) $=$ "remote" benign vascular sexual and exertional headache as defined in text.

Patients were classified into those experiencing benign vascular sexual headache alone, and those experiencing both benign vascular sexual headache and benign exertional headache. The latter group was reclassified according to whether the exertional headache had occurred within 72 hours of the sexual headache (close), or more than 72 hours apart (remote).

\section{Results}

The records of 45 patients with benign vascular sexual headache were reviewed. Thirty nine patients were first seen in private rooms and six patients were first seen in the hospital emergency department. Twenty seven $(60 \%)$ of the headache patients had experienced benign vascular sexual headache alone and 18 $(40 \%)$ had experienced both benign vascular sexual headache and benign exertional headache. The mean age, age range, and sex distribution are shown in table 1 for all patient groups. Patients followed up were representative of the original sample in terms of age and sex distribution (Students $t$ test and normal approximation to the binomial distribution, respectively), with 17 of them experiencing benign vascular sexual headache alone and 13 of them experiencing both benign vascular sexual headache and benign exertional headache.

The following information was obtained at follow up: 1) The mean (SD) duration of follow up was $74(51.7)$ months with a range of 9-236 months; 2) A history of migraine was found in $\mathbf{4 7 \%}$ of patients and a family history in $30 \%$ of patients (table 2); 3) All patients experienced their sexual headaches whilst undertaking an "active" role (as defined by the patient) in sexual activity; 4) Patients had experienced from one to innumerable benign vascular sexual headaches and/or benign exertional headaches before initial review.

Benign vascular sexual headache alone patients (17 patients)

On specific questioning at follow up, $59 \%$ (10/17) of the benign vascular sexual headache patients could identify stress and/or fatigue as a contributing factor to their initial headache. 2) Following diagnosis $41 \%(7 / 17)$ had recurrences and two of these patients experienced their headache recurrences in distinct clusters.

Case 1 was a 29 year old woman who had had 10 attacks of benign vascular sexual headache over a four week period. She abstained from further sexual activity for a two month period until the diagnosis was made. Following reassurance and a two week course of propranolol (40 $\mathrm{mg}$ daily), she resumed sexual activity. The headaches had occurred at times of significant financial and social stress which had resolved by the time she resumed sexual activity. Three years later she had three further attacks of benign vascular sexual headache over a one week period at a time of similar stresses to the first occasion. She abstained until the financial and social pressures had eased and then resumed sexual activity without further headache.

Case 2 was a 42 year old man who developed his sexual headaches, after returning from a prolonged remote posting, with four headaches over a one week period. He responded to reassurance. This occurred once again 27 months later under similar circumstances.

The other patients who experienced recurrences after diagnosis included, a 49 year old man with four episodes over two years of

Table 2 Migraine history and duration of follow up of review cases

\begin{tabular}{llll}
\hline Review cases & Personal Hx migraine & Family $H x$ migraine & Duration of follow up (months) \\
\hline BVSH+/-BEH & $14 / 30(47 \%)$ & $9 / 30(30 \%)$ & $74.0(51 \cdot 7)$ \\
BVSH alone & $8 / 17(47 \%)$ & $5 / 17(29 \%)$ & $71 \cdot 3(56 \cdot 1)$ \\
BVSH/BEH & $3 / 9(33 \%)$ & $2 / 9(22 \%)$ & $65.4(48.1)$ \\
BVSH/BEH (close) & $3 / 4(75 \%)$ & $2 / 4(50 \%)$ & $104.8(37.7)$ \\
BVSH/BEH (remote) & &
\end{tabular}

Legend: BVSH = benign vascular sexual headache, BEH = benign exertional headache, BVSH/BEH (close) = "close" benign vascular sexual and exertional headache and BVSH/BEH (remote) $=$ "remote" benign vascular sexual and exertional headache as vascular sexual
defined in text. 
follow up, a 45 year old man with two episodes over a three year period and three patients with ongoing benign vascular sexual headache who had intermittently experienced these headaches for 116, 121, and 236 months, at the follow up review. These patients did not need any medical therapy for the headaches. Two of them related the recurrences to exessive fatigue and/or stress, and by avoiding sexual activity at these times have significantly reduced their frequency.

Of the seven patients who experienced recurrences after diagnosis, six of them identified stress and/or fatigue as a precipitant of their recurrent headache.

All patients received an explanation of their condition and reassurance at their initial review. Seven patients were also treated with beta blockade (propranolol in six patients at $40-80 \mathrm{mg} / \mathrm{day}$, and atenolol in one patient at a dose of $50 \mathrm{mg} /$ day), for two to eight weeks. This treatment was associated with no further headaches in six patients whilst taking the medication, and two patients with ongoing benign vascular sexual headache had a reduced frequency of headache, but elected to discontinue the medication after recognising the precipitating factors (stress and/or fatigue). Reassurance, beta blockade, and diazepam (in three patients taken before sexual activity) appeared effective, although many patients took the latter two medications for a short period, with no recurrence on stopping. The relative merits of these treatments is uncertain, however, most patients elected not to take or remain on medication after receiving reassurance and an explanation on the benign nature of their sexual headache.

Benign vascular sexual and exertional headache patients (13 patients)

Patients were classified into two well defined groups;

1) The first group experienced their (vascular) sexual and exertional headaches within 72 hours of each other ("close" benign vascular sexual and exertional headaches) (8/9 patients within 48 hours and $5 / 9$ patients within 24 hours.) Either sexual (4/9) or exertional (5/9) headache occurred first. The headache was often linked by a residual headache, which was typically mild, constant, and generalised. The severity and length of the benign vascular sexual headache and benign exertional headache were usually variable although the headaches were of a similar quality and distribution. Recurrences occurred in 1/9 patients with "close" benign vascular sexual and exertional headaches when he experienced a single exertional headache eight years later (with a 10 year follow up period from presentation). Another patient, a 37 year old man, recalled at follow up a similar benign vascular sexual headache 20 years previously when orgasm occurred in the standing position.

2) The second group experienced their headaches separated by more than 72 hours ("remote" benign vascular sexual and exertional headaches). These four patients experienced their sexual and exertional headaches separated by between one month (in a patient with recurrent benign exertional headache), and several years. Recurrences appeared in 2/4 patients with "remote" benign vascular sexual and exertional headaches with both patients (a 26 year old female and a 20 year old male) experiencing recurrent exertional headache. Both patients have adapted their lifestyle to avoid further headache, which in one patient proved unresponsive to propranolol therapy. Reassurance has been the main method of treatment with propranolol proving effective in 4/5 patients in which it was used. Two of the patients have coped with recurrent exertional headache by lifestyle modification, and the other 11 have not required further treatment for recurrences.

Five of the thirteen patients experienced their exertional headache whilst undertaking isometric exercise such as screaming, singing or performing a valsalva manoeuvre and a further five during isotonic exercise such as running or hurrying up a few steps. The remainder were participating in exercise that involved both isotonic and isometric activity (three). There was no association between the type of exercise and other variables.

\section{Discussion}

A subgroup of patients with benign vascular sexual headache who also experience benign exertional headache has been identified in this study. Previous reports of patients experiencing both benign vascular sexual headache and benign exertional headache are scarce, although two patients described in this series have been previously reported, ${ }^{45}$ and Powell ${ }^{6}$ reported a weight lifter who experienced an exertional headache whilst doing leg presses and then four days later experienced a vascular sexual headache. In Lance's ${ }^{2}$ original series of 21 patients with type 1 and 2 benign sexual headache, two patients with benign vascular sexual headache also experienced exertional headaches, but these were of a different quality, as were the exertional headaches in the patient described by Paulson and Klawans. ${ }^{3}$

Lance also described one patient with type 1 benign sexual headache whilst undertaking a passive role in the sexual act, ${ }^{2}$ but this patient cannot be compared directly with the benign vascular sexual headache patients in this study. It is important when attempting to identify any association between benign sexual headache and benign exertional headache that one subdivides benign sexual headache according to the presumed pathophysiology. In this study, the patients experiencing benign vascular sexual headache and benign exertional headache all experienced sudden onset of a sustained vascular headache during the relevant activity. Hence, it is reasonable in some of these patients to interpret that the sexual activity represents a form of exertion with additional autonomic changes that occur with orgasm. ${ }^{8}$

Further support for this concept comes from the work of Masters and Johnson, ${ }^{9}$ and others, ${ }^{10}{ }^{11}$ on the physiological changes associated 
with coitus. They have documented rises in heart rate, systolic and diastolic blood pressure approaching those that may be achieved with exercise, particularly in younger patients around the time of orgasm. Perhaps sexual activity should be viewed more as a vigorous form of physical exertion, which in many patients is their major form of physical exertion. This is in keeping with the recognised association between the unfit and overweight patient and sexual headaches. ${ }^{13}$ It seems likely that both benign vascular sexual headache and benign exertional headache, in some circumstances share a common pathophysiological basis, possibly related to haemodynamic changes that occur at the time of orgasm with stretching of pain sensitive intracranial structures.

Patients experiencing benign vascular sexual headache and to a lesser extent, benign vascular sexual headache and benign exertional headache together showed a strong male predominance (table 1). This is in contrast to the female prepdominance found in migraine patients and may in some circumstances relate to the degree of physical exertion in the sexual act. In this series of benign vascular sexual headache patients, a past history of migraine was found in $47 \%$ of patients and a family history in $30 \%$. Previous reviews of benign sexual headache have found a lower prevalence of migraine $\mathrm{Johns}^{14}$ in his literature review found $23 \%$ of patients with benign sexual headache had a past history of migraine, and $11 \%$ a family history, although this included patients with types 1 and 3 benign sexual headache, in addition to benign vascular sexual headache. Interestingly, many authors have proposed that benign vascular sexual headache is a migraine variant, ${ }^{314} 15$ because of the clinical similarity,,$^{14}$ efficacy of beta blockade, ${ }^{15}$ and more recent description of familial occurrence of vascular sexual headache. ${ }^{14}$ Alternatively, benign vascular sexual headache patients may share the same vascular hyperreactivity that has been described in migraine patients, ${ }^{16}$ increasing the likelihood of their concurrence. Further studies of cerebral blood flow, platelet aggregation, serotonin, and vascular hyperreactivity may clarify this point.

Vascular hyperreactivity may also in some way relate to the priming effect that was seen in many of the patients with benign vascular sexual headache alone and also the "close" benign vascular sexual and exertional headache patients. Many of these patients experienced several vascular sexual headaches with or without exertional headache over a period of days to weeks, with the headaches often linked by a mild background headache. The resulting therapeutic implications leads us to advise our patients to abstain from sexual activity and active physical exertion for an empirical four day period following a sexual headache, or until the residual headache has completely resolved. In addition, any obvious precipitating factors such as stress or fatigue should have been recognised, if not resolved before sexual activity is resumed. In many cases the patient will enforce sexual or exertional abstinence through fear of recurrence or persisting dis- comfort, but without appropriate advice the potential exists for development of psychosexual problems.

Benign vascular sexual headache and benign exertional headache with their sudden onset may in many ways mimick a small subarachnoid haemorrhage. In studies of patients with subarachnoid haemorrhage $3 \cdot 8-12 \%$ occur during sexual intercourse. ${ }^{17} 18$ These can often be distinguished from benign sexual headache by the absence of previous sexual headaches, alteration of consciousness, longer duration of the headache, and signs of meningeal irritation including neck stiffness (and low back pain and sciatica in the ambulant patient). In patients in whom the clinical differentiation is unclear a cranial CT and CSF examination may be required. The "sentinel headache" of subarachnoid haemorrhage may prove more difficult to distinguish from benign sexual or exertional headache, in those cases in which it is due to stretching or haemorrhage into the aneurysm wall rather than to a minor leak into the subarachnoid space. ${ }^{19} 20$

The paroxysmal nature of benign vascular sexual headache extends beyond the association with stress and fatigue as noted in this study. It is not known why benign vascular sexual headache (and also benign exertional headache) occur with such variable patterns. Not surprisingly therefore $41 \%$ of patients with benign vascular sexual headache experienced recurrences from months to years after initial presentation and diagnosis, and six of these seven patients recalled stress or fatigue as being a major factor at the time of recurrence. As expected this is similar to the findings of Rooke ${ }^{12}$ who in his study of 93 patients with benign exertional headache found that almost two thirds of patients were still experiencing their headache after five years. The only previous prognostic study into benign sexual headache was that of Lance $^{2}$ who found that after a follow up period of two to seven years (of patients with type 1 and 2 benign sexual headache) four of the 21 patients were still experiencing occasional sexual headaches. Wijdick ${ }^{21}$ in his study of 71 patients with thunderclap headache included three patients with benign vascular sexual headache and 12 patients with exertional headache. It is, however, unclear whether the sexual or exertional headache patients were represented in those with recurrences.

Propranolol has been described as an effective therapy for benign vascular sexual headache. ${ }^{15}$ Many of our patients, however, responded to reassurance and explanation. When beta blockade was prescribed, a short course (two to eight weeks) seemed adequate, with recurrences not being directly related to stopping. Whilst the beta blockade may be important for its effects on the haemodynamic state or vascular hyperreactivity, other more acute factors (including stress and fatigue in a predisposed patient) must play a role. Indomethacin is the recognised treatment for benign exertional headache but was not evaluated in this study. ${ }^{22}$

There were no identifiable factors to differentiate the three groups of patients (ben- 
ign vascular sexual headaches alone, "close" benign vascular sexual and exertional headaches, and remote benign vascular sexual and exertional headaches) other than the priming effect that appeared to exist within the first two groups. Further studies of the pathophysiology of benign vascular sexual headache and its relationship with exertional headache are needed.

1 Kriz K. Coitus as a factor in the pathogenesis of neurological complications. Cesk Neurol Neurochir 1970;33:162-7.

2 Lance JW. Headaches related to sexual activity. $J$ Neurol Neurosurg Psychiatry 1976;39:1226-30.

3 Paulson GW, Klawans HL. Benign orgasmic cephalgia. Headache 1974;13:181-7.

4 Edis RH, Silbert PL. Sequential benign sexual headache and exertional headache. Letter. Lancet 1988;i:993.

5 Silbert PL, Hankey GJ, Prentice DA, AP Simon HT. Angiographically demonstrated arterial spasm in a case of Angiographically demonstrated arterial spasm in a case of benign sexual headache and beeni
Aust NZJ Med 1989;19:466-8.

6 Powell B. Weight lifters cephalgia. Ann Emerg Med 1982;11:449-51.

7 Headache classification committee of the International Headache Society. Classification and diagnostic criteria for headache disorders, cranial neuralgias and facial pain. Cephalalgia 1988;8 (supp 7):19-25.

8 Braun A, Klawans HL. Headaches associated with exercise and sexual activity. In: F Clifford Rose, ed. Handbook of clinical neurology. Amsterdam: Elsevier, 1986:373-82.

9 Masters WH, Johnson VE. Human sexual response. Boston: Little, Brown, 1966:291.

10 Bartlett RG, Jr. Physiologic responses during coitus. J Appl Physiol 1956;9:469-72.

11 Hellerstein HK, Friedman EH. Sexual activity and the postcoronary patient. Arch Intern Med 1970;125:987-99.

12 postcoronary patient. Arch Intern Med 1970;125:987-99. 1968;52/4:801-8.

13 Paulson GW. Headaches associated with orgasm. Med Asp Hum Sex 1975;9:7-16.

14 Johns DR. Benign sexual headache within a family. Arch Neurol 1986;43:1158-60.

15 Porter M, Jankovic J. Benign coital cephalalgia. Differential diagnosis and treatment. Arch Neurol 1981;38:710-2.

16 Drummond PD, Lance JW. Neurovascular disturbances in headache patients. Clin Exp Neurol 1984;20:93-99.

17 Locksley HB. Natural history of subarachnoid haemorrhage, intracranial aneurysms and arteriovenous malformations, based on 6368 cases in the cooperative study. J Neurosurg 1966;25:219-39.

18 Lundberg PO, Osterman PO. The benign and malignant forms of orgasmic cephalgia. Headache 1974;14:164-5.

19 Leblanc $R$. The minor leak preceding subarachnoid haemorrhage. J Neurosurg 1987;66:35-9.

20 Verweij RD, Wijdicks EFM, van Gijn J. Warning headache in aneurysmal subarachnoid haemorrhage. A case controlled study. Arch Neurol 1988;45:1019-20.

21 Wijdicks EFM, Kerkhoff H, van Gijn J. Long-term follow up of 71 patients with thunderclap headache mimicking subarachnoid haemorrhage. Lancet 1988;i:68-70.

22 Diamond S. Prolonged benign exertional headache: Its clinical characteristics and response to Indomethacin. Headache 1982;22:96-8. 